\title{
An Error-reduction Mothod for Temporal Phase Unwrapping Based on Double Three-step Phase-shifting and Least-squares Fitting
}

\author{
Liu Yong ${ }^{1, ~ a ~, ~ H u a n g ~ D i n g f a ~}{ }^{2, b}$ and Jiang Yong ${ }^{1, c}$ \\ ${ }^{1}$ School of Computer Science and Technology, Southwest University of Science and Technology \\ Mian Yang, China \\ ${ }^{2}$ School of Civil Engineering, Southwest Jiaotong University \\ Cheng Du, China \\ aliuyong@swust.edu.cn, ${ }^{b}$ dfhuang@home.swjtu.edu.cn, 'randomjy@126.com
}

Keywords: phase-shifting interferometry; temporal phase unwrapping; heterodyne principle; least-squares fitting

\begin{abstract}
Phase-shifting interferometry on structured light projection is widely used in 3-D surface measurement. An investigation shows that least-squares fitting can significantly decrease random error by incorporating data from the intermediate phase values, but it cannot completely eliminate nonlinear error. This paper proposes an error-reduction method based on double three-step phase-shifting algorithm and least-squares fitting, and applies it on the temporal phase unwrapping algorithm using three-frequency heterodyne principle. Theoretical analyses and experiment results show that this method can greatly save data acquisition time and improve the precision.
\end{abstract}

\section{Introduction}

Phase unwrapping is a main research content in interferometry measurement. It can be classified into two categories of spatial phase unwrapping (SPU) and temporal phase unwrapping (TPU). SPU algorithms are based on the comparison of neighboring pixels in 2D space so that it is difficult in practice to tackle object surfaces with complicated geometry and topology because of phase ambiguity and error propagation. TPU algorithms consider interference phase at a given pixel $\Phi$ as a function of time $t$, so the one-dimensional unwrapping along the time axis can avoid the path-dependence problems. Comparing with SPU, TPU algorithms have significant improvements on reliability, accuracy, and computation time[1,2].

A lot of classical TPU algorithms have been presented in the past two decades, including linear sequence TPU (LTPU)[1], exponential sequence TPU (ETPU)[3], reversed exponential sequence TPU (RETPU)[4] and three-frequency heterodyne principle TPU (TFHTPU)[5]. In addition, some modified TPU algorithms has been known, such as: a simple version of TPU[6], a generalized reversed exponential sequence TPU[7], a modified TPU[8], a generalized TPU[9], a simplified exponential sequence TPU algorithm (SETPU)[10], etc.

For the purpose of decreasing random error by incorporating data from the intermediate phase values, an investigation was carried out by Huntley and Saldner[4], in which least-squares fitting was used to three classical TPUs (LTPU, ETPU and RETPU). Results showed that least-squares fitting can significantly reduce random errors on the condition that the data acquisition time and reliability of original TPU algorithm remain unchanged.

Related researches[11-13] showed that there are two main error sources, electronic noise and nonlinearity, in the shape measurement system using the projected fringe technique. It is obvious that the nonlinear errors from camera or projector have been neglected in Huntley's investigation. The effects of nonlinear errors cannot be totally ignored, though four-step phase-shifting can provide better immunity from nonlinearities than three-step phase-shifting and the intensity of nonlinear errors is relatively smaller than that of random errors. Considering this problem, a double three-step phase-shifting (DTPS) algorithm was proposed by Huang[13], in which three-step phase shifting is done twice with an initial phase offset of $60^{\circ}$ between them, and the two obtained phase maps are 
averaged to generate the final phase map. Both theoretical and experimental results showed that this algorithm worked well in significantly reducing the measurement error and saving data acquisition time.

An investigation on error-reduction method for TFHTPU based on DTPS and least-squares fitting is carried out in this paper. The rest of paper is organized as follows: Section 2 analyses nonlinear errors existed in four-step phase-shifting algorithm and its effects on error-reduction methods with least-squares fitting. Section 3 presents the error-reduction method for TFHTPU. Section 4 analyses and compares the errors of classical TPU algorithms. Section 5 shows the experiment results. The last section summarizes the main results of this work.

\section{Nonlinear errors analysis of current error-reduction methods with least-squares fitting}

Nonlinear errors existed in Phase-shifting interferometry (PSI) have been well examined through experimentation and by computer simulation for a long time[14]. As we know now, over-exposure [11] of the image sensor array and the gamma[12] of the projector are two main reasons of nonlinear errors. As proved by Huang[13], there exists mainly third-order phase error caused by nonlinearities of both the camera and the projector in three-step phase-shifting. The similar result can be proved that there exists mainly forth-order phase error in four-step phase-shifting. Generally, the intensity of third-order phase error is far greater than that of fourth-order phase error, so four-step phase-shifting can provide better immunity from nonlinearities than three-step phase-shifting. That's why the nonlinear errors were ignored in the investigations by Huntley[4].

To investigate the effects of nonlinear errors on error-reduction methods with least-squares fitting, nonlinear errors corresponding to every fringe density of method D[4] are shown in Fig.1. It's clear that nonlinear errors at an identical pixel are separately corresponding to different real phase values, some are positive and others are negative. With least-squares fitting, they are added up on separate weighting factors. The distributions of nonlinear errors in method B and D[4] can be simulated and shown in Fig.2. It's obvious that the nonlinear phase error of final phase value is greatly reduced, but some peaks are obviously remained at some special real phase values. Although increasing the value of $N$ in $N$-step phase-shifting algorithm can provide increasing immunity from nonlinearities[11], greater $N$ than four will lead to the data acquisition time of TPU greatly increased.

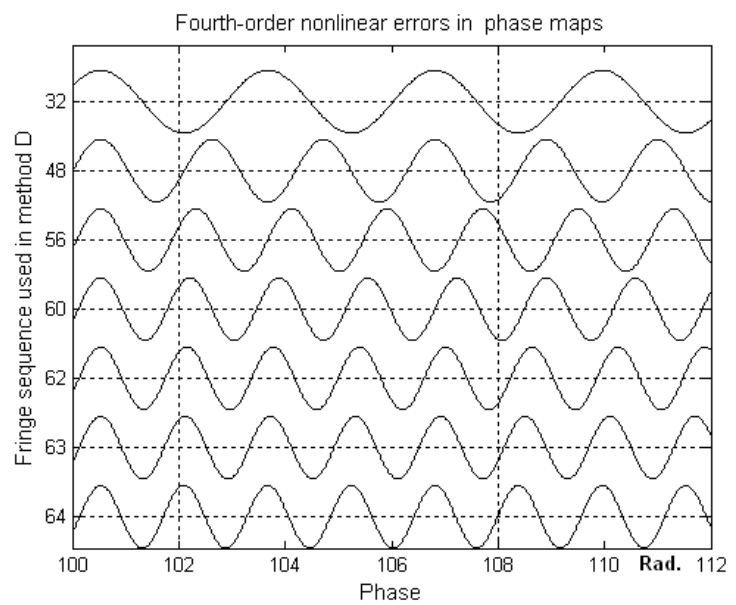

Fig. 1 Four-order nonlinearity errors of unwrapped phase maps in reversed exponential sequence $(s=64)$.
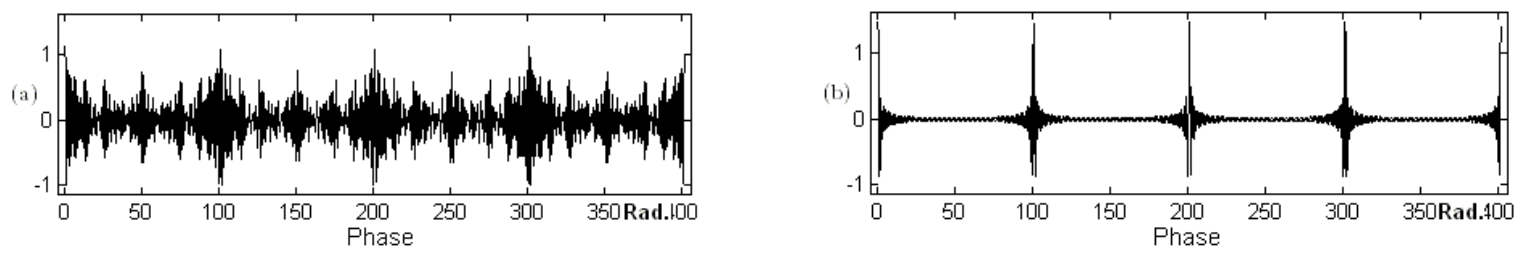

Fig.2 The distributions of nonlinear error in method B and D[4].

(a)method D; (b)method B 


\section{Error-reduction method for TFHTPU}

Double three-step phase-shifting algorithm[13] provides us a simple and feasible approach to decrease nonlinear errors effectively. As described in Ref.13, by increase an additional fringe sequence (with an initial phase offset of $60^{\circ}$ ) on a certain TPU algorithm, the third-order phase errors can be cancelled out by average operator.

Take TFHTPU for example, the fringe sequence in original TFHTPU with three-step phase-shifting can be written by

$$
t=59,64,70
$$

Wrapped phase map corresponding to each $t$ in Eq.(1) can be computed by

$$
\phi_{t}=\arctan \left[\sqrt{3} \frac{I_{1, t}-I_{3, t}}{2 I_{2, t}-\left(I_{1, t}+I_{3, t}\right)}\right],
$$

where $I_{i, t}(i=1,2,3)$ are captured three images at time point $t$.

According to DTPS, another fringe sequence with an initial phase offset of $60^{\circ}$ in each time point of Eq.(1) should be captured, and wrapped phase maps should be computed by Eq.(2), too. In order to distinguish the two set of wrapped phase maps, $\phi_{t}$ and $\phi_{t}{ }^{\prime}$ are used to denote the two sets of wrapped phase maps, respectively.

By unwrapping all wrapped phase maps, we can get six unwrapped phase maps, which can be described by

$$
\Phi(t)=U\left[\phi_{59}, \phi_{64}, \phi_{70}\right] \quad t=59,64,70
$$

and

$$
\Phi^{\prime}(t)=U\left[\phi_{59}{ }^{\prime}, \phi_{64}{ }^{\prime}, \phi_{70}{ }^{\prime}\right]-\pi / 3 \quad t=59,64,70
$$

, respectively, where $U[$.$] denotes the phase unwrapping operation by TFHTPU algorithm.$

Then, three intermediate phase maps can be calculated by

$$
\bar{\Phi}(t)=\left(\Phi(t)+\Phi^{\prime}(t)\right) / 2 \quad t=59,64,70
$$

Now, least-squares fitting can be used upon $\bar{\Phi}(t)$ to reduce measurement error and compute the final phase value at identical $s$. For the convenience of comparison between current error-reduction methods, $s=64$ is used in the following descriptions.

\section{Precision analysis and comparison}

To compare the accuracy between current error-reduction methods, five TPU algorithms will be addressed below: exponential sequence without fitting (method A), fitted to linear sequence (method B), fitted to exponential sequence $(\operatorname{method} C)$, fitted to reversed exponential sequence (method D) and fitted TFHTPU with DTPS (method E).

Random errors analysis of methods A-D has been given by Huntley and Saldner[4]. If there are additive and Gaussian errors in the measured phase values, the measured sequence of unwrapped phase values can be written as

$$
\Phi(t)=\omega t+\varepsilon_{\phi},
$$

where $\varepsilon_{\phi}$ is a Gaussian random variable, with a mean of zero and a standard deviation of $\sigma_{\phi} \cdot \omega$ represents the rate of change of phase with time $t$.

The estimator $\hat{\omega}$ of $\omega$ in method $\mathrm{A}$ is given by

$$
\hat{\omega}_{A}=\Phi(s) / s
$$


with the intermediate phase values playing no part in the calculation. The standard deviation of the random variable $\hat{\omega}_{A}$ follows directly from Eq. (7) as

$$
\sigma_{A}=\sigma_{\phi} / s
$$

In method B, all phase values are unwrapped and used for the fitting (a fitting in the least-squares sense of the line), the estimator $\hat{\omega}$ of $\omega$ in method $\mathrm{B}$ is given by

$$
\hat{\omega}_{B}=\sum_{t=1}^{s} t \Phi(t) / \sum_{t=1}^{s} t^{2} .
$$

The standard deviation of the random variable $\hat{\omega}$ follows directly from Eq. (9) as

$$
\sigma_{B}=\frac{\sqrt{6} \sigma_{\phi}}{[s(s+1)(2 s+1)]^{1 / 2}} \approx \sqrt{3} \sigma_{\phi} / s^{3 / 2} .
$$

In method $\mathrm{C}$ and method $\mathrm{D}$, the standard deviation of the random variable $\hat{\omega}$ can be given by

$$
\sigma_{C}=\frac{\sqrt{3} \sigma_{\phi}}{\left(4 s^{2}-1\right)^{1 / 2}} \approx \sqrt{3} \sigma_{\phi} /(2 s)
$$

and

$$
\sigma_{D}=\frac{\sigma_{\phi}}{\left[s^{2}\left(\log _{2} s-2 / 3\right)+2 s-(1 / 3)\right]^{1 / 2}} \approx \sigma_{\phi} /\left(s \sqrt{\log _{2} s}\right) \quad \text { for } s \gg 1 .
$$

As described in previous section, least-squares fitting can be used upon $\bar{\Phi}(t)$ (Eq.(5)) in method E. From Eq.(9) of Ref.4 we know that $\Phi(t)$ is a linear function of time $t$. In theory, we can compute the phase value $\tilde{\Phi}(s)$ at identical fringe density $(t=s)$ from any unwrapped intermediate phase value $\bar{\Phi}(t)$ by a transform as

$$
\tilde{\Phi}(s)=\bar{\Phi}(t) \times s / t=\left(\omega t+\varepsilon_{\phi}\right) \times s / t=\omega s+\varepsilon_{\phi} \times s / t .
$$

Comparing $\tilde{\Phi}(s)$ in Eq.(13) with the unwrapped phase value $\bar{\Phi}(s)$ in Eq.(5), the phase error of $\tilde{\Phi}(s)$ is enlarged $s / t$ times than that of $\bar{\Phi}(s)$. The error ratio of $s / t$ in Eq.(13) is so closed to 1 that we can safely assume that the errors of $\tilde{\Phi}(s)$ and $\bar{\Phi}(s)$ are taken from the same Gaussian distribution. Under the transform of Eq.(13), the estimator $\hat{\omega}$ of $\omega$ in method E can be written as

$$
\hat{\omega}_{E}=\sum_{t} t \bar{\Phi}(t) / \sum_{t} t^{2} \quad(t=64,59,70) \quad \approx 3 \bar{\Phi}(s) / s .
$$

In spite of that, statistical averaging is used to reduce the errors in Eq.(5). As we know that statistical averaging can be used to reduce the random errors by $\sqrt{n}$, where $n$ is the number of sample data in one operation, the standard deviation of the random variable $\hat{\omega}_{E}$ can be given by

$$
\sigma_{E}=\sigma_{\phi} /(\sqrt{6} s)
$$

\section{Experiments}

The fringes and phase-shifts were generated by computer, and then were projected by a digital projector (SONY VSP-EL7) onto a flat plate which is employed as the measurement object. A video camera (NIKON MVC1000SAM-GE30ST) with resolution of $1280 \times 1024$ pixels was used to acquire encoded fringe patterns. The fringes were horizontal; $s=64$ was used in methods A-E. 
The measured errors of methods A-E were shown in Fig. 3 and RMS errors were given in Table 1. To verify the precisions of error-reduction methods (B-E), errors ratios (error $\sigma_{\phi}$ in method A to error $\sigma$ in other methods) and theoretical error ratios were calculated and given in Table 1, too.
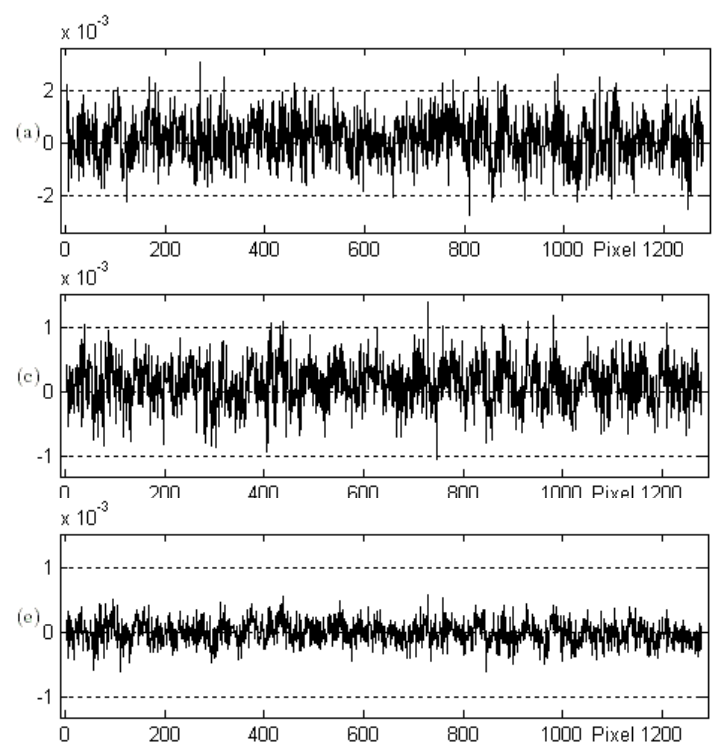

Fig.3 Phase errors in methods A-E. (a) A; (b) C; (c) D; (d) E; (e) B.
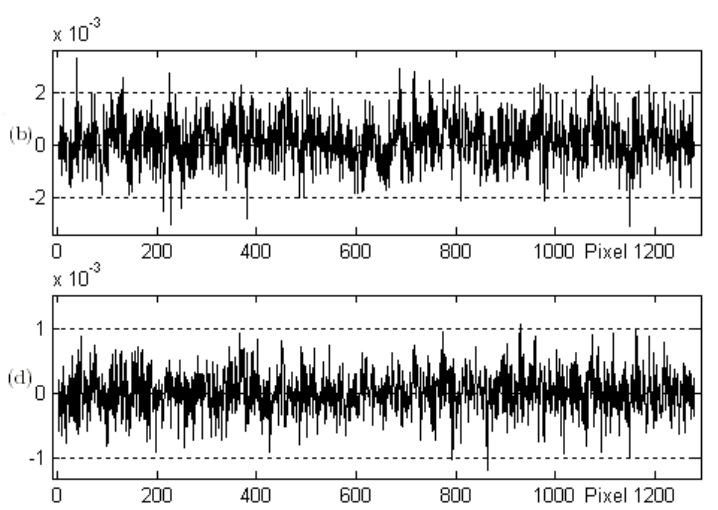

Table 1 RMS errors and error ratios in method A-E

\begin{tabular}{cccccc}
\hline Method & $\mathrm{A}$ & $\mathrm{B}$ & $\mathrm{C}$ & $\mathrm{D}$ & $\mathrm{E}$ \\
\hline RMS error $\left(\times 10^{-4}\right)$ & 8.3292 & 1.7997 & 7.3906 & 3.3970 & 3.3194 \\
error ratio $\left(\sigma_{\phi} / \sigma\right)$ & 1 & 4.628 & 1.127 & 2.452 & 2.635 \\
theoretical error ratio & 1 & 4.619 & 1.155 & 2.828 & 2.449 \\
\hline
\end{tabular}

It can be clear seen from Fig.3 that: compared with the error distributions of method D (Fig.3(c)) and method B (Fig.3(e)), that of method E (Fig.3(d)) is more even along the field of view, and some peaks from the nonlinear errors are significantly weakened. The effectiveness of method $\mathrm{E}$ can be further verified from Table 1: RMS error of method $\mathrm{E}$ is lower than that of method $\mathrm{C}$, while the number of captured images used in the former is shorter than that used in the later, as we know that the total number of captured images is 18(6 fringe densities, 3 frames for each fringe density) in method E vs. 28(7 fringe densities, 4 frames for each fringe density) in method C. Furthermore, RMS error ratio is greater than theoretical error ratio in method $\mathrm{E}$, while it's the opposite in methods $\mathrm{C}$ and $\mathrm{D}$. This result shows that the nonlinear errors have greater influence on the measurement precision of method $\mathrm{C}$ and D.

\section{Summary}

Accuracy, data acquisition time, computation time and reliability are four main evaluation indexes for temporal phase unwrapping algorithms. Theoretical analyses and experiments show that the nonlinear errors existed in current error-reduction methods can be inhibited well in this method. Furthermore, this method keeps higher precision, smaller data acquisition time and computation time comparing to the method $\mathrm{C}$ (fitted to exponential sequence), though the latter is considered as the most appropriate method to situations in which the emphasis is on achieving a maximum measurement speed with reasonable accuracy[4]. 


\section{References}

[1] J.M. Huntley and H.O. Saldner, Temporal phase-unwrapping algorithm for automated interferogram analysis, Appl. Opt. 32 (1993) 3047-3052.

[2] H.O. Saldner and J.M. Huntley, Temporal phase unwrapping: application to surface profiling of discontinuous objects, Appl. Opt. 36(1997) 2770-2775.

[3] J.M. Huntley, H.O. Saldner, Shape measurement by temporal phase unwrapping: comparison of unwrapping algorithms, J. Meas. Sci. Technol. 8(1997) 986- 992.

[4] J.M. Huntley, H.O. Saldner, Error-reduction methods for shape measurement by temporal phase unwrapping, J. Opt. Soc. Am. A 14(1997) 3188-3196.

[5] Carsten Reich, Reinhold Ritter, Jan Thesing, White light heterodyne principle for 3D-measurement, SPIE 3100(1997) 236-224.

[6] H. Zhao, W.Y. Chen, Y.S. Tan, Phase-unwrapping algorithm for the measurement of three-dimensional object shapes, Appl. Opt. 33(1994) 4497-4500.

[7] L. Kinell, M. Sjodahl, Robustness of reduced temporal phase unwrapping in the measurement of shape, Appl. Opt. 40(2001) 2297-2303.

[8] X. Peng, Z. Yang, and H. Niu, Multi-resolution reconstruction of 3D image with modified temporal unwrapping algorithm, Opt. Commun. 224(2003) 35-44.

[9] J. D. Tian, X. Peng, X. B. Zhao, A generalized temporal phase unwrapping algorithm for three-dimensional profilometry, Optics and Lasers in Engineering 46(2008) 336-342.

[10]Z.H. Xu , X.Y. Su, An algorithm of temporal phase unwrapping, Journal of Sichuan University (Natural Science Edition) 45(2008) 537-540.

[11]C. R. Coggrave, J. M. Huntley, Optimization of a shape measurement system based on spatial light modulators, Opt. Eng. 39(2000) 91-98.

[12] Georg H. Notni and G. Notni, Digital fringe projection in 3D shape measurement: an error analysis, Proc. SPIE 5144(2003) 372-380.

[13]P. S. Huang, Q.Y. J. Hu, and F.P. Chiang, Double three-step phase-shifting algorithm, Appl. Opt. 41(2002) 4503-4508.

[14]J. Schmit, K. Creath, Extended averaging technique for derivation of error-compensating algorithms in phase-shifting interferometry, Appl. Opt. 34(1995) 3610-3619. 